\title{
Feasibility of exposure response prevention to treat repetitive behaviors of children with autism and an intellectual disability: A brief report
}

\author{
Brian A. Boyd, \\ Division of Occupational Science and Occupational Therapy, University of North Carolina at \\ Chapel Hill, Chapel Hill, North Carolina, USA \\ Cooper R. Woodard, and \\ Groden Center, Providence, Rhode Island, USA \\ James W. Bodfish \\ Carolina Institute for Developmental Disabilities, University of North Carolina at Chapel Hill, \\ Chapel Hill, North Carolina, USA
}

\begin{abstract}
There is a lack of evidence-based behavioral therapies or pharmacotherapies to treat repetitive behaviors found in autism. Effective behavioral therapies are needed to counter any negative consequences these behaviors may have on the child's early learning and socialization. The purpose of this proof-of-principle study was to test the feasibility of modifying exposure response prevention, an evidence-based strategy for obsessive-compulsive disorder, to treat the repetitive behaviors found in autism. Five school-aged participants (ages 5-11) diagnosed with an autism spectrum disorder participated in the study. Our preliminary findings suggest it is feasible, and potentially efficacious, to modify standard exposure response prevention to treat the specific forms of repetitive behaviors found in individuals with autism and comorbid intellectual disabilities. A larger clinical trial is needed to substantiate these preliminary findings.
\end{abstract}

\section{Keywords}

autism; exposure response prevention; repetitive behaviors; treatment

Researchers have found a variety of discrete types of repetitive behaviors that are expressed clinically in autism (Lam and Aman, 2007). This broad range of behavior has been subdivided into two conceptual categories: 'lower-order' motor actions (stereotyped movements, repetitive manipulation of objects, and repetitive forms of self-injurious behavior) that are characterized by repetition of movement, and more complex or 'higherorder' cognitive behaviors (compulsions, rituals and routines, insistence on sameness, and circumscribed interests) that are characterized by a rigid adherence to some rule or mental set (e.g. needing to have things 'just so') (Turner, 1999). Both categories of behavior appear to be a function of an overall behavioral rigidity/lack of flexibility, whereby situations that involve lack of or limited access to particular rituals or routines can trigger bouts of severe

\footnotetext{
(C) The Author(s) 2011

Corresponding author: Brian A. Boyd, Division of Occupational Science and Occupational Therapy, Department of Allied Health Sciences, University of North Carolina at Chapel Hill, 2050 Bondurant Hall, CB\# 7122, Chapel Hill, NC 27599-7120, USA, brian_boyd@med.unc.edu.

Reprints and permissions: sagepub.co.uk/journalsPermissions.nav
} 
irritability, aggression, self-injury, or other repetitive and problem behaviors (Green et al., 2006).

Because obsessive-compulsive disorder (OCD) and autism appear to share at least some phenomenological and pathogenic similarities, it is reasonable to ask if psychosocial treatments for repetitive behaviors in OCD also would be effective in treating repetitive behaviors found in autism (Rapaport and Inoff-Germain, 2000). On purely phenomenological grounds, both OCD and autism involve both behavioral manifestations (e.g. compulsions in OCD; rituals/routines in autism) and cognitive manifestations (e.g. obsessions in OCD; insistence on sameness and preoccupations in autism), although the form or the content of these symptoms differs in the two disorders (McDougle et al., 1995; Zandt et al., 2007), with autism tending to involve less complex forms that is perhaps due to co-occurring cognitive and/or language impairments. Despite any overlap, the profile of repetitive behaviors in autism and OCD is also differentiable (Bejerot, 2007). For example, individuals with OCD typically do not exhibit the repetitive motor behaviors often associated with autism (e.g. hand flapping). Additionally, individuals with OCD exhibit more cleaning, checking, and counting behaviors, whereas individuals with autism engage in more hoarding, ordering, touching/tapping, and self-injury (McDougle et al., 1995). In OCD, it is presumed that repetitive actions (compulsions) function to avoid negative mood states (e.g. anxiety), however, some types of repetitive behavior in autism seem to be associated with approach-motivation or positive mood states (e.g. unusual interests, preoccupations). In examining pathophysiology, similarities in neurochemical function have been noted between OCD and autism, including serotonin and dopamine systems, and this information has guided drug treatment research efforts. Thus far, the use of drugs to treat repetitive behaviors in autism has proved less successful (King et al., 2009) than in OCD. Similarly, it is not clear that behavioral treatments designed for OCD could be directly translated to address the specific forms of repetitive behavior that occur in autism.

Exposure and response prevention (ERP) is an evidence-based intervention strategy that has been used as part of a broader cognitive-behavior therapy (CBT) approach (Huppert and Franklin, 2005). ERP is often combined with other CBT-based strategies such as relaxation training. The exposure component of ERP typically involves the repeated, gradual exposure of the individual to environmental stimuli associated with symptoms of anxiety and the subsequent expression of compulsive behaviors. The response prevention component involves the active avoidance of the compulsive act. ERP, itself, is based on the behavioral extinction paradigm that anxiety attenuates after repeated exposure to the anxiety/distressproducing stimulus and repeated prevention of compulsive behavior associated with that stimulus.

Given the apparent efficacy of ERP in the treatment of OCD, it appears to be a reasonable candidate for investigation as a treatment of repetitive behaviors in autism. In two previously reported case studies of a CBT/ERP-based approach for children with autism (Lehmkuhl et al., 2008; Reaven and Hepburn, 2003), modified forms of ERP were used to treat comorbid OCD symptoms in autism. Both of the aforementioned case studies involved children with autism who did not have co-occurring cognitive deficits. As a near majority of children with autism manifest comorbid intellectual disabilities it is important to determine if an ERP-based approach also can be modified to treat repetitive behaviors in the context of lower-functioning autism. This study was designed to be an initial proof-of-principle study to examine the feasibility of modifying ERP to treat repetitive behaviors characteristic of autism in a group of children with co-occurring cognitive deficits. 


\section{Method}

\section{Participants}

Five children with a diagnosis of autism spectrum disorder (ASD) participated in this study. All participants attended a residential school for children with developmental disabilities and behavioral disorders located in a medium-sized city in northeastern United States.

Participants ranged in age from five to 11 years $(M=8.6)$ and all had comorbid intellectual disabilities based on psychological testing data from school records. Diagnostic status was confirmed by a clinical psychologist using Diagnostic and Statistical Manual of Mental Disorders (DSM)-IV criteria; in addition, teachers completed the Social Communication Questionnaire (SCQ; Rutter et al., 2003). The SCQ is a valid ASD screening tool; the lifetime version of this measure provides cut-off scores for ASD (score of $\geq 15$ ) and autism (score of 222). See Table 1 for participant demographic information. Participants had to meet the following inclusion criteria to be enrolled in this feasibility study: (a) between five to 18 years of age, (b) previous diagnosis of an ASD, (c) informed consent obtained from child's guardian and/or child assent to participate in the study, and (d) clinically significant level of repetitive behaviors as determined by a baseline score of 2 or greater on the 'frequency' or 'accommodation' subscales of the Inventory for Repetitive Behavior (IRB) (structured clinical interview; Bodfish, 2003); or a total score of $\geq 20$ on the Repetitive Behavior Scales-Revised (RBS-R) (psychometrically validated informant report measure; Bodfish et al., 1999). Exclusion criteria included an unstable medical illness or initiation of another formal treatment for repetitive behaviors (medication and/or behavioral treatment) within the previous three months.

\section{Treatment procedures}

Prior to the start of treatment, enrolled children entered a two-week baseline phase to determine pre-treatment level and severity of repetitive behaviors (see Table 2) that involved a combination of classroom observation by research staff and teacher completion of the IRB. Study participants received two treatment sessions per day during the treatment phase. The total treatment phase lasted for a period of two weeks. Treatment sessions were conducted in a therapy room in the school that was separate from the child's classroom. The room contained the participant-specific repetitive behavior trigger stimuli, mastered academic task materials, a table, two chairs, and a small, video monitor suspended from the ceiling. A trained behavioral therapist conducted all treatment sessions. Each session lasted approximately 15 to 20 minutes. Following the two-week treatment session, each child's teacher was trained in the procedure, and conducted the same frequency and duration (i.e. 15-20 minutes) of sessions in the classroom setting. Three months after these classroom sessions terminated, the therapist conducted a one-week set of 'booster' sessions with the child in the therapy room.

ERP treatment condition-The ERP treatment consisted of alternating discrete trials of the child having access to items that elicit repetitive behaviors with trials of academic instruction tasks. This alternating, discrete trial format was designed to provide the child with explicit exposures to the trigger stimuli (i.e. the exposure component of ERP), and to provide the child with verbal instructions on stopping rituals (e.g. 'Now, it's time to do some work.') on a predetermined schedule (i.e. the response prevention component of ERP). We included an academic task for children to engage with during the ERP trials because of their cognitive capacity, which necessitated the use of concrete, specific tasks for children to complete to maintain their focus and engagement. Importantly, this was done while the stimuli that cued the ritual remained visible in an attempt to maximize the exposure part of ERP. During the ERP sessions, the children received eight to 10 discrete trials of the following therapy sequence: (a) allow ritual/restricted behavior (on a variable interval 
schedule that averaged one minute, with no verbal praise or reprimands provided for engaging in appropriate or inappropriate behavior), (b) interrupt repetitive behavior (using no more than a light physical redirection or touch, which was used as needed across sessions) but leave trigger items in child's presence, (c) administer academic task (on a variable interval schedule that averaged one minute, with brief verbal praise for staying on task), and (d) interrupt academic task. The therapist wore an earpiece that provided an audio cue for the randomly generated intervals.

\section{Outcome measures}

Behavioral coding-A direct observational coding system was used to measure four specific participant outcomes: (a) percentage of time engaged in a competing response (academic tasks), (b) amount of time that elapsed before the participant physically touched the repetitive behavior trigger item (i.e. latency), (c) amount of time that elapsed before the participant actually began to engage in repetitive behavior, and (d) frequency of cooccurring problem behavior (e.g. aggression, tantrums). Behavioral raters, naïve to the purposes of the study, coded the videotapes post-hoc. Reliability between raters for the videotaped observation data was calculated in Observer 5.0 (Noldus, 1991), a behavioral coding software program that allows for real time coding of frequency and duration-based behaviors. Outcome data for behavioral observations are reported as a standardized change score by comparing the mean of the participants first two ERP sessions with the mean of their last two sessions.

Teacher ratings-Teacher ratings were completed to determine if treatment effects seen in the therapy setting generalized to the child's classroom. Teacher ratings also provide some external validation of treatment outcomes, as teachers remained naïve to the nature of the therapeutic intervention for the period that the child was receiving intervention in the therapy room. We calculated teacher ratings by summing scores across four subscales of a project-developed rating scale that was based on the IRB measure - frequency (i.e. how often the behavior occurs), interference (i.e. whether the behavior interferes with school/ other activities), intensity (i.e. level of distress shown when the restricted interest/ritual is blocked), and accommodation (i.e. how often items are provided to the child to allow him to engage in the behavior). For each participant, two teacher ratings were completed one week apart prior to the start of sessions for baseline purposes. The same teacher again completed the rating at the end of the two-week ERP treatment. Finally, three months after the research sessions had ended, teacher ratings were completed at the end of a set of 'booster' sessions that were conducted by the therapist.

\section{Results}

Results are based on a combination of direct observational data and teacher ratings.

\section{Reliability of behavioral observations}

Two independent behavioral raters coded $40 \%$ of videotaped observations to establish interrater reliability. Reliability for the duration codes (Academic Engagement, RB Item Available, RB Engagement) was calculated in Observer using a duration/sequence based comparison method. Inter-rater reliability for duration codes was $96.6 \%$. Reliability for problem behavior, the only frequency code, was calculated using a frequency/sequence based comparison method using a two-second tolerance window (to be counted as an agreement, independent raters had to independently 'turn on' the behavior code within this window of time). Inter-rater reliability for this sole frequency code was $55.6 \%$. Reliability for problem behavior was lower due to its infrequent occurrence. 
Outcomes for behavioral observations-On average, the percentage of time ERP participants engaged with the academic task increased from the start of treatment (i.e. the first two sessions) $(M=41.2 \%$, range $=32-47 \%)$ to the end of treatment (i.e. the last two sessions) $(M=52.8 \%$, range $=37-67 \%)$ (see Table 3 ). In addition, the amount of time (measured in seconds) participants delayed their engagement with the repetitive behavior stimulus increased over the intervention period (treatment start $M=0.4 \mathrm{~s}$, range $=0.0-1.95$ $\mathrm{s}$; treatment end $M=12.1 \mathrm{~s}$, range $=0.0-30.5 \mathrm{~s}$ ). Similarly, the average latency time before the participant began to physically express repetitive behavior increased from the start of treatment $(M=2.1 \mathrm{~s}$, range $=0.0-4.7 \mathrm{~s})$ to the end of treatment $(M=35.7 \mathrm{~s}$, range $=0.0$ $100.7 \mathrm{~s})$. Finally, the rate of problem behavior decreased over the course of the intervention period (treatment start $M=0.3$, range $=0.0-1.0$; treatment end $M=0.06$, range $=0.0-0.15$ )

Outcomes for teacher ratings-Teacher ratings for severity of repetitive behavior based on summing frequency + interference + intensity + accommodation were completed at the end of the second week of the baseline period, at the end of ERP treatment, and at the end of the one-week 'booster' session (see Table 4). The averaged summed score across the five participants was 9.0 (range $=6-14)$ for the baseline period, $7.2($ range $=2-12)$ for the ERP period, and 6.2 (range $=3-10$ ) for the booster period. The mean change score from baseline to the end of ERP treatment was $21.4 \%$ (range $=-16-66 \%$ ), and the mean change score from baseline to the end of the booster session was $32.8 \%$ (range $=20-50 \%$ ).

\section{Discussion}

This initial proof-of-principle study suggests standard ERP can be modified to treat more autism-specific forms of repetitive behaviors found in individuals with autism and cooccurring cognitive deficits. Two previous case studies reported positive effects in using CBT/ERP to treat the co-occurring OCD symptoms of individuals with autism (Reaven and Hepburn, 2003; Lehmkuhl et al., 2008); however, the children in these case studies had intact cognitive abilities. The current study modified ERP to use with children with autism who have comorbid intellectual disabilities, and to treat the associated autism-specific forms of repetitive behavior. Generally, convergent positive changes were found for the direct observational data and teacher ratings. However, the outcomes found were modest, perhaps due to the acute nature of the treatment trial (i.e. two weeks in duration). This finding may suggest that an increased dosage is needed to achieve larger treatment effects either in terms of intensity or duration of treatment. Yet, the teacher ratings imply that sustained effects are possible as four of five participants maintained or increased the amount of change teachers reported between the baseline phase and a three-month follow-up period.

With the increasing numbers of children with autism being placed in school settings, it is imperative that novel treatments either be directly implemented in these settings, or at least show generalization of treatment gains to children's classrooms. We found that it was feasible to implement a modified version of ERP in a school-based context. Although initially a trained behavioral therapist conducted the therapy sessions, classroom teachers were successfully trained to implement the intervention in the interim period between the end of the two-week ERP treatment phase and the collection of three-month follow-up data. Even if clinically significant levels of repetitive behaviors are found it is not clear from the current findings that ERP is always the most appropriate form of treatment. Thus, the question becomes for what type of repetitive behaviors is this treatment most appropriate? Based on our preliminary work, this treatment may be most effective when there are identifiable and tangible antecedent stimuli (e.g. toy cars) that reliably predict the onset of the child's repetitive behavior (e.g. lining up the cars) to allow repeated opportunities for ERP trials to occur. Further, ERP may prove useful in helping to provide an empirical 
rationale to use medications to treat repetitive behaviors, in particular for children, if these individuals fail to respond to intensive behavioral therapy.

The small sample size limits the generalizability of any study findings; however, there are some clinical and research implications that can be deduced. First, the use of multiple outcome measures allowed us to detect modest changes in repetitive behavior over the course of an acute treatment period. Second, it appears that other forms of treatment for repetitive behavior are needed to augment effects for those participants who are nonresponders. Finally, given that repetitive behaviors first begin to manifest in the preschool years of children with autism, ERP may be useful as an early, intensive behavioral treatment to prevent any long-term, negative consequences that result from engaging in behaviors that interfere with learning and socialization. Future research should examine the effectiveness of treatments targeted at the two classes of repetitive behaviors (i.e. lower order and higher order) to determine if differential treatment approaches are needed. For this study, ERP was mostly used to treat lower forms of repetitive behavior. In addition, previous research has suggested that increases in adaptive behaviors (e.g. social skills) are associated with decreases in the occurrence of repetitive behaviors (Boyd et al., in press). Thus, future clinical trials could compare direct interventions specifically designed to decrease repetitive behaviors in autism with indirect interventions that target other core symptoms, yet may have collateral effects on repetitive behaviors.

\section{References}

Bejerot S. An autistic dimension. Autism. 2007; 11:101-110. [PubMed: 17353211]

Bodfish, JW. Unpublished Rating Scale. University of North Carolina-Chapel Hill, Western Carolina Center Research Reports; 2003. Inventory for Repetitive Behaviors (IRB).

Bodfish, JW.; Symons, FJ.; Lewis, MH. The Repetitive Behavior Scales (RBS). Chapel Hill, NC: Western Carolina Center Research Reports; 1999.

Boyd BA, McDonough SM, Bodfish JW. Evidence-based behavioural interventions for repetitive behaviours in autism. Journal of Autism and Developmental Disorders. in press.

Green V, Sigafoos J, Pituch K, Itchon J, O'Reilly M, Lancioni G. Assessing behavioural flexibility in individuals with developmental disabilities. Focus on Autism and Other Developmental Disabilities. 2006; 21:230-236.

Huppert JD, Franklin ME. Cognitive behaviour therapy for obsessive compulsive disorder: An update. Current Psychiatry Reports. 2005; 7:268-273. [PubMed: 16098280]

King B, Hollander E, Sikich K, McCraken J, Scahill L, Bregman J, et al. Lack of efficacy of citalopram in children with autism spectrum disorders and high levels of repetitive behavior. Archives of General Psychiatry. 2009; 66:583-590. [PubMed: 19487623]

Lam KSL, Aman MG. The repetitive behavior scale - revised: Independent validation in individuals with autism spectrum disorders. Journal of Autism and Developmental Disorders. 2007; 37:855866. [PubMed: 17048092]

Lehmkuhl H, Storch EA, Bodfish JW, Geffken GR. Brief report: Exposure and response prevention for obsessive compulsive disorder in a 12-year-old with autism. Journal of Autism and Developmental Disorders. 2008; 38:977-981. [PubMed: 17885801]

McDougle CJ, Kresch LE, Goodman WK, Naylor ST, Volkmar FR, Cohen DJ, Price LH. A casecontrolled study of repetitive thoughts and behaviour in adults with autistic disorder and obsessive-compulsive disorder. American Journal of Psychiatry. 1995; 152:772-777. [PubMed: 7726318]

Noldus, LPJJ. The Observer. Wageningen, Netherlands: Noldus Information Technology; 1991.

Reaven J, Hepburn S. Cognitive-behavioural treatment of obsessive-compulsive disorder in a child with Asperger syndrome. Autism. 2000; 7(2):145-164. [PubMed: 12846384] 
Rapaport JL, Inoff-Germain G. Practitioner review: Treatment of obsessive-compulsive disorder in children and adolescents. Journal of Child Psychology and Psychiatry. 2000; 41:419-431. [PubMed: 10836672]

Rutter, M.; Bailey, A.; Lord, C. Social Communication Questionnaire. Los Angeles, CA: Western Psychological Services; 2003.

Turner M. Annotation: Repetitive behaviour in autism: A review of psychological research. Journal of Child Psychology and Psychiatry. 1999; 40:839-849. [PubMed: 10509879]

Zandt F, Prior M, Kyrios M. Repetitive behaviour in children with high functioning autism and obsessive compulsive disorder. Journal of Autism and Developmental Disorders. 2007; 37:251259. [PubMed: 16865546] 


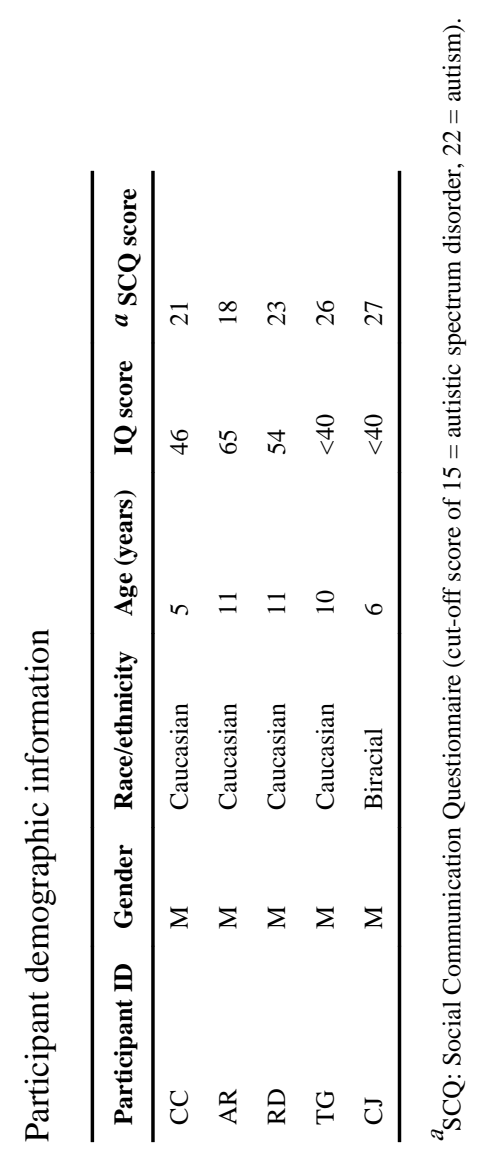

Autism. Author manuscript; available in PMC 2013 July 14. 
Table 2

Severity and type of repetitive behaviors

\begin{tabular}{lll}
\hline Participant ID & RBS-R score & Type of repetitive behavior \\
\hline CC & 30 & Preoccupation with timers, watches, clocks that have digital or illuminated displays \\
AR & 19 & Preoccupation with Nintendo Gameboy (one specific game) \\
RD & 21 & Completeness, must complete all activities \\
TG & 32 & Preoccupation/verbal ritual about Disney movies and specific sections of select movies \\
CJ & 25 & Must empty/dump-out containers \\
\hline
\end{tabular}

RBS-R: Repetitive Behavior Scales-Revised (higher scores indicate greater severity). 


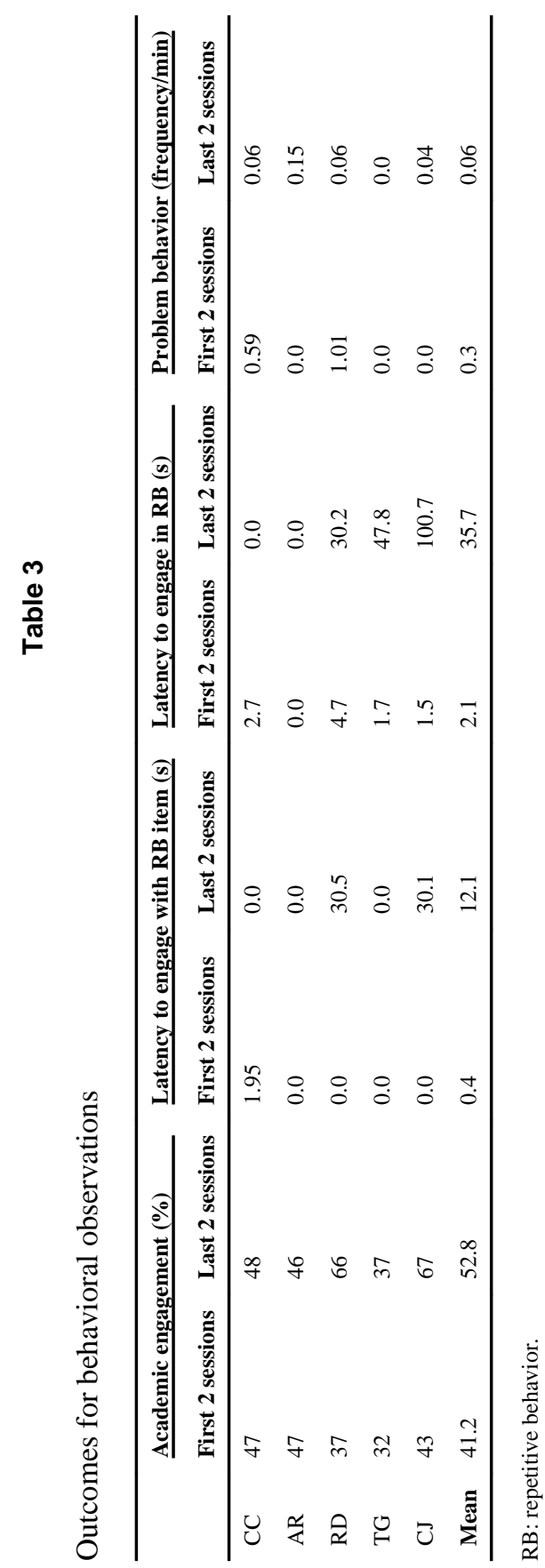

Autism. Author manuscript; available in PMC 2013 July 14. 


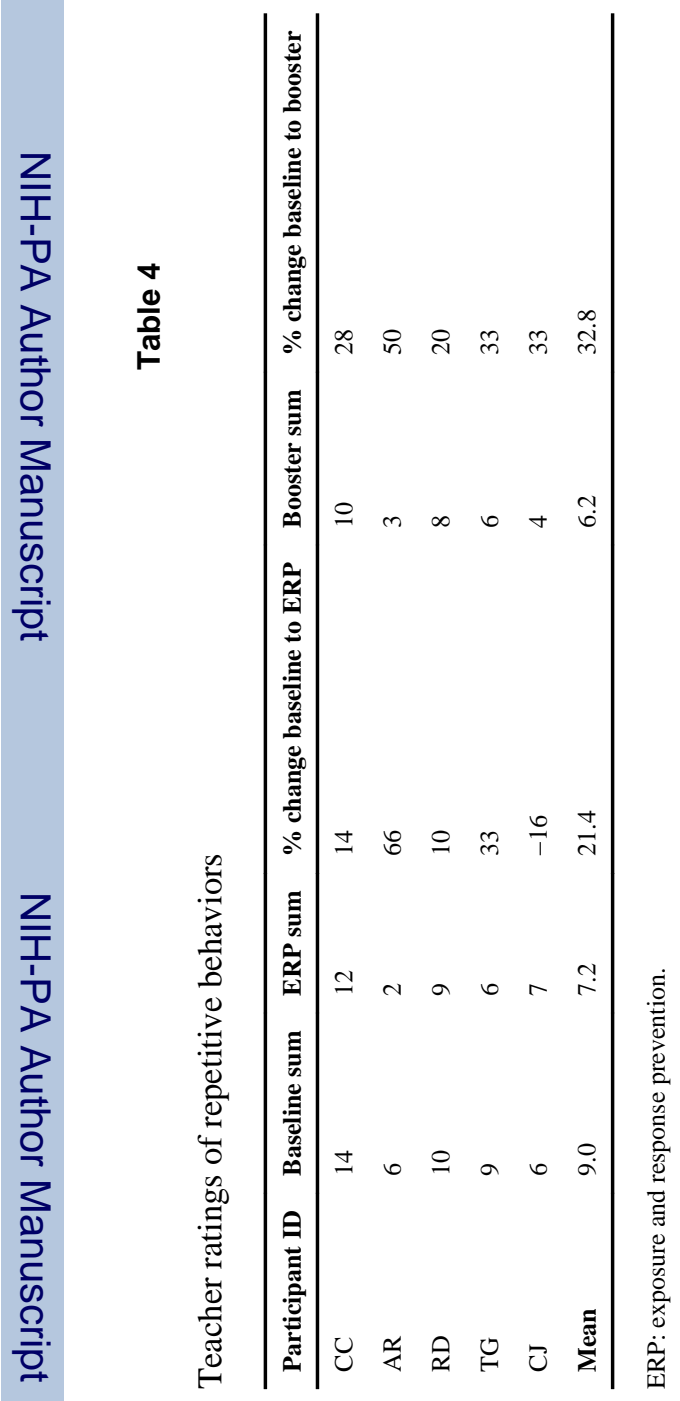

Autism. Author manuscript; available in PMC 2013 July 14. 